\title{
Ibuprofen Use Did Not Affect Outcome Metrics After Arthroscopic Rotator Cuff Repair,
}

\author{
Nicholas R. Kraus, M.S., B.A., Kirsten D. Garvey, M.S., B.A., \\ Laurence D. Higgins, M.D., M.B.A., and Elizabeth Matzkin, M.D.
}

\begin{abstract}
Purpose: To determine whether patients who are prescribed ibuprofen after arthroscopic rotator cuff repair have significantly different patient-reported outcomes for pain, function, and overall health at baseline and 1 and 2 years after operation relative to patients only prescribed opioids. Methods: Patients who underwent a rotator cuff repair by a total of 3 surgeons and participated in the outcomes registry from 2012 to 2016 were screened for inclusion in this study. Inclusion criteria were primary arthroscopic rotator cuff repair, at least 2 years from the date of surgery and over the age of 18. Exclusion criteria were revision and open rotator cuff repair. All patients followed the standard postoperative rehabilitation protocol for rotator cuff repair. Patients were divided into 2 cohorts. Group I included patients who received ibuprofen/nonsteroidal anti-inflammatory agents (NSAID) after surgery $(\mathrm{n}=281)$, and Group II consisted of patients who did not receive ibuprofen/NSAID after surgery $(n=182)$. Patient-reported outcome measures for Visual Analogue Scale, American Shoulder Elbow Surgeons score, Single Assessment Numeric Evaluation score, Simple Shoulder Test and The Veterans Rand 12-Item Health Survey were collected preoperatively and at 3 and 6 months, 1 year, and 2 years after surgery. Statistical analysis was performed to compare patient-reported outcome measures between Group I and II. Results: This study consisted of 463 patients who underwent arthroscopic rotator cuff repair, and patients were divided into 2 cohorts. There were 281 patients who did not receive ibuprofen/NSAID after operation in Group I and 182 patients who did receive ibuprofen in Group II. There were no statistically significant differences between the 2 groups in age at treatment, mean body mass index, gender, ethnicity, diabetes, and number of rotator cuff tendons involved; however, there was a statistically significant difference in receiving worker's compensation $(P=.005)$, and this was subsequently adjusted for in our analysis. There were no significant differences in patient-reported outcomes for all metrics between the group prescribed ibuprofen and the group that was not prescribed ibuprofen at 1 and 2 years after surgery or in change from baseline. Conclusion: Patients receiving ibuprofen did as well as patients who did not receive ibuprofen after arthroscopic rotator cuff repair on patient-reported outcome measures assessing shoulder pain, function, and overall health. Level of Evidence: Level III, retrospective comparative study.
\end{abstract}

$\mathbf{R}$ otator cuff injury is one of the most widespread musculoskeletal disorders, accounting for more than 4.5 million physician visits and 250,000 surgeries

From Tufts University School of Medicine (N.R.K., K.D.G.) and Department of Orthopaedic Surgery, Brigham and Women's Hospital (N.R.K., K.D.G., L.D.H., E.M.), Boston, Massachusetts.

The authors report the following potential conflicts of interest or sources of funding: L.D.H reports personal fees from Arthrex, Inc., outside the submitted work. Full ICMJE author disclosure forms are available for this article online, as supplementary material.

Received April 6, 2020; accepted November 15, 2020.

Address correspondence to Elizabeth Matzkin, M.D., Harvard Medical School,75 Francis St., Boston, MA 02115.E-mail: ematzkin@bwh.harvard. edu

(C) 2020 THE AUTHORS. Published by Elsevier Inc. on behalf of the Arthroscopy Association of North America. This is an open access article under the CC BY-NC-ND license (http://creativecommons.org/licenses/by-nc-nd/4.0/). 2666-061X/20434

https://doi.org/10.1016/j.asmr.2020.11.004 per year in the United States. ${ }^{1-4}$ Injury to the rotator cuff often causes shoulder pain, loss of strength, complex sequelae, and partial or total inability to work, thus reducing a patient's quality of life..$^{5-8}$

The treatment algorithm for rotator cuff tears is multifactorial. ${ }^{9}$ The rotator cuff has a limited ability for intrinsic repair, in part due to poor vascularization of the tendon tissue., 2,10,11 Management depends on the presence and severity of impingement, extent of tendon tear, degree of retraction, and functional demands. ${ }^{12}$ Nonoperative treatment can lead to tear progression, tendon retraction, and muscle degeneration. ${ }^{11}$ Surgical rotator cuff repair has demonstrated satisfactory longterm clinical results, ${ }^{13}$ with more than $90 \%$ good or excellent results at 10 years after surgery. ${ }^{14}$

The ideal rotator cuff repair restores kinematics, improves strength and function while decreasing pain. ${ }^{15,16}$ Despite advances in both open and arthroscopic surgical 
techniques, shoulder surgery is frequently associated with severe postoperative pain. ${ }^{17,18}$ Traditional pain management for shoulder injuries has included local injection of analgesics, nerve blocks, intravenous patient-controlled analgesia, opioid drugs, and nonsteroidal antiinflammatory agents (NSAIDs), with the latter two being the most frequently used. ${ }^{19,20}$ Despite the widespread use of opioids and NSAIDs in the postoperative setting, both have risks, limitations, and side effects.

Opioids are associated with adverse effects including nausea and vomiting, pruritus, sleep disturbance, constipation, and dependency. ${ }^{21}$ Conversely, NSAIDs function by inhibiting cyclooxygenase (COX) enzymes, reducing the synthesis of inflammatory prostaglandins $^{22}$ but diminishing platelet counts, leading to bleeding and gastric ulceration. ${ }^{23}$ Although NSAIDs have been shown to minimize pain and decrease opioid requirements after surgery, ${ }^{24}$ they have traditionally been avoided by orthopedic surgeons. This avoidance could be based on past studies that have shown that selective NSAIDs (COX-2 inhibitors) impede fracture and tendon healing. ${ }^{25-28}$ Additionally, in vitro NSAID treatment has been shown to impede proliferation and migration of tendon cells that are necessary for tendon repair after injury. ${ }^{29}$ Despite literature indicating NSAID use leads to adverse in vitro and clinical outcomes, other studies have shown neutral or positive effects of selective and nonselective NSAID use. . $^{30-33}$

Given the widespread use of NSAIDs for postoperative pain relief, it is important to identify what effects NSAID use has on patient-reported outcomes for pain, function, and overall health after arthroscopic rotator cuff repair. There is limited information regarding the direct effect of NSAIDs on clinical and patient-reported outcomes in rotator cuff repair. ${ }^{34}$ The purpose of this study is to determine whether patients that are prescribed ibuprofen after arthroscopic rotator cuff repair have significantly different patient-reported outcomes for pain, function, and overall health at baseline and 1 and 2 years after surgery relative to patients only prescribed opioids. We hypothesized that patients who were prescribed ibuprofen after arthroscopic rotator cuff repair would not have significantly different patient-reported outcome measures as those that were instructed not to take ibuprofen after surgery.

\section{Methods}

Approval by the institutional review board was obtained before the initiation of the present study. Data were collected prospectively on consecutive surgeries performed by 3 surgeons at an academic medical center and retrospectively reviewed for the present study. All patients signed an informed consent. An electronic surgical outcomes registry was used for data collection. Patients who underwent a rotator cuff repair and participated in the outcomes registry from 2012 to 2016 were screened on the basis of inclusion and exclusion criteria reviewed. Inclusion criteria were primary arthroscopic rotator cuff repair during the study period, at least 2 years from the date of surgery, and age over 18 years. Exclusion criteria were revision rotator cuff repair and open rotator cuff repair. All patients followed the standard postoperative rehabilitation protocol for rotator cuff repair.

Patients included in the study were divided into two cohorts based on post-operative analgesic therapy: Group I (no ibuprofen/NSAID use) and Group II (ibuprofen use). Group I patients were instructed to avoid ibuprofen/NSAIDs after surgery for 12 weeks and were provided opioids for pain management. Group II patients were sent home with a prescription for $800 \mathrm{mg}$ of Ibuprofen and instructed to take regularly and use opioid pain medication when needed. The groups were predetermined by surgeon preference; all patients undergoing surgery with a particular surgeon either were prescribed ibuprofen or were instructed to avoid ibuprofen/NSAIDs for 3 months after surgery.

All participants were administered a preoperative survey consisting of the following outcome-measuring tools: (i) Visual Analog Scale (VAS) used to measure overall pain level, (ii) the Veterans Rand 12-Item Health Survey (VR-12), a standard self-reported global health measure tool that is used to assess a patient's overall perspective of their health, (iii) the American Shoulder and Elbow Surgeons Shoulder Score (ASES) used to measure functional limitations and pain of the shoulder, (iv) standard preoperative form consisting of 4 questions regarding their expectations of recovery, (v) Single Assessment Numeric Evaluation (SANE) shoulder score used to determine a patient self-assessment of their shoulder function, and (vi) Simple Shoulder Test used to assess functional disability of the shoulder based on a 12-item score card. The same outcome measures were reassessed at 3 and 6 months and 1 and 2 years after surgery. At each data collection point, participants were e-mailed an electronic survey with one e-mail reminder and one phone call reminder by a research assistant if outcome measures were not completed in a timely manner. Paperbased questionnaires were not used in this study.

\section{Statistical Analysis}

The scores for all functional assessment metrics were tallied for each cohort at each of the time points of data collection pre- and post-operatively. Baseline differences in covariates were assessed using the $t$-test (continuous) and $\chi^{2}$ test (categorical variables). A linear mixed effects model to compare outcomes between the groups at each timepoint accounted for all available data and did not require that subjects have complete data at all timepoints. Multivariable models adjusted for covariates that were imbalanced at baseline (worker's compensation status). Statistical analysis was performed to determine 
Table 1. Patient Demographics

\begin{tabular}{|c|c|c|c|}
\hline Demographic characteristics & No ibuprofen/NSAIDS Use (281 patients) & Ibuprofen use (182 patients) & $P$ value \\
\hline Age at treatment (mean \pm SD) & $57.01 \pm 9.13$ & $55.93 \pm 9.72$ & .229 \\
\hline BMI, median (IQR) & $28(22.14-33.86)$ & $27.90(20.8-35.0)$ & .805 \\
\hline Gender & & & .157 \\
\hline Females ( $\%$ of total) & $117(42 \%)$ & $89(49 \%)$ & \\
\hline Missing & 2 & 0 & \\
\hline Ethnicity & & & .525 \\
\hline Non-Hispanic Black ( $\%$ of total) & $8(3 \%)$ & $2(1 \%)$ & \\
\hline Other ( $\%$ of total) & $38(14 \%)$ & $29(16 \%)$ & \\
\hline Missing & 7 & 3 & \\
\hline Diabetic & & & .465 \\
\hline No $(\%$ of total $)$ & $269(96 \%)$ & $172(95 \%)$ & \\
\hline Yes ( $\%$ of total) & $10(4 \%)$ & $9(5 \%)$ & \\
\hline Missing & 2 & 3 & \\
\hline Number of tendons involved & & & .897 \\
\hline 1 & $82(42.3 \%)$ & $81(44.5 \%)$ & \\
\hline 2 & $77(39.7 \%)$ & $72(39.6 \%)$ & \\
\hline 3 & $31(16.0)$ & $27(14.8 \%)$ & \\
\hline 4 & $4(2.1 \%)$ & $2(1.1 \%)$ & \\
\hline Missing & 89 & 6 & \\
\hline
\end{tabular}

BMI, body mass index; IQR, interquartile range, NSAID, nonsteroidal anti-inflammatory agent; SD, standard deviation.

whether there was any correlation between ibuprofen use and functional outcome measures collected. Statistical significance was set at $P<.05$.

\section{Results}

\section{Patient Characteristics}

In total, 591 patients undergoing primary rotator cuff repair were initially identified. Of these patients, 471 were included $(80 \%)$, and 120 did not have complete preoperative and 1- and 2-year postoperative PROMs data $(20 \%)$ and were therefore excluded. For patients who underwent multiple surgeries, only the first surgery was included, leaving an analytic dataset of 463 .
Differences in baseline characteristics between the included subjects are presented in Table 1. There were no statistically significant differences between the 2 groups in terms of age at treatment, mean body mass index, gender, ethnicity, and diabetes; however, there was a statistically significant difference between both groups with regard to worker's compensation status $(P=.005)$.

There was a total of 281 patients in Group I (no NSAIDs use) and 182 patients in Group II (ibuprofen/ NSAID use) who underwent rotator cuff repair during the data collection period. These patients were administered the preoperative and postoperative surveys at each of the study time points. Clinical characteristics of

Table 2. Improvement in VAS Pain Ratings Across All Groups After Rotator Cuff Repair (Adjusted for Worker's Compensation Status) and in ASES Pain Ratings Across All Groups After Rotator Cuff Repair (Adjusted for Worker's Compensation Status)

\begin{tabular}{|c|c|c|c|c|}
\hline PRO & Time & No ibuprofen/NSAIDs use & Ibuprofen use & $P$ value \\
\hline$\overline{\text { VAS }}$ & Baseline & $5.0(4.6,5.4)$ & $5.4(5.0,5.8)$ & .112 \\
\hline VAS & Year 1 & $1.4(1.1,1.7)$ & $1.6(1.3,1.9)$ & .279 \\
\hline VAS & Year 2 & $1.2(0.9,1.5)$ & $1.3(1.0,1.6)$ & .629 \\
\hline VAS & Year 2 change from BL & $-3.8(-4.2,-3.4)$ & $-4.1(-4.5,-3.7)$ & .274 \\
\hline ASESI & Baseline & $47.1(44.0,50.2)$ & $44.0(41.0,47.1)$ & .110 \\
\hline ASESI & Year 1 & $83.3(80.6,85.9)$ & $82.4(79.9,84.9)$ & .559 \\
\hline ASESI & Year 2 change from BL & $38.5(35.5,41.5)$ & $41.9(38.5,45.3)$ & .142 \\
\hline
\end{tabular}

BL, baseline; ASES, American Shoulder and Elbow Surgeons Shoulder; NSAID, nonsteroidal anti-inflammatory agent; VAS, Visual Analog Scale. 
Table 3. Improvement in SANE Ratings Across All Groups After Rotator Cuff Repair (Adjusted For Worker's Compensation Status) And In SST Ratings Across All Groups After Rotator Cuff Repair (Adjusted For Worker's Compensation Status)

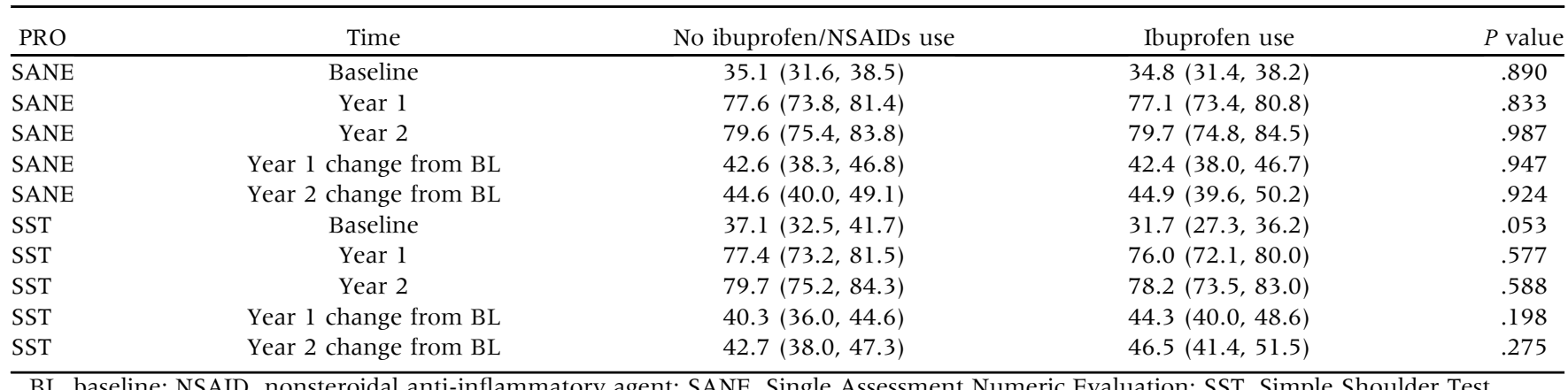

BL, baseline; NSAID, nonsteroidal anti-inflammatory agent; SANE, Single Assessment Numeric Evaluation; SST, Simple Shoulder Test.

each group were assessed to evaluate major differences in rotator cuff tear size by comparing number of tendons torn. There were no statistical differences between the 2 NSAID use groups in terms of the number of tendons involved.

\section{Postoperative Pain}

Table 2 presents the VAS pain scores after adjusting for worker's compensation status. Patients experienced significant improvement in VAS pain scores at 1 - and 2years after rotator cuff repair in both Group I (no NSAID use) and Group II (ibuprofen/NSAID use). Patients in Group I had an average baseline VAS pain score of 5.0 (confidence interval [CI] 4.6-5.4), a 1-year pain score of 1.4 (CI 1.1-1.7), and a 2-year pain score of 1.2 (CI 0.9-1.5). Patients in Group II had an average baseline VAS pain score of 5.4 (CI 5.4-5.8), a 1-year pain score of 1.6 (CI 1.3-1.9) and a 2-year pain score of 1.3 (CI 1.0-1.6). Both Group I and Group II postoperative VAS pain scores meet the Minimal Clinically Important Differences measure. There is no significant difference in VAS pain score between patients in group I and group II at 1 year $(P=.279)$ and 2 years after operation $(P=.629)$. Patients across both groups demonstrated a similar magnitude of pain improvement at 1 and 2 years after rotator cuff repair.
Pain improvement was also measured with the ASES assessment tool. After adjusting for worker's compensation status, there were no statistically significant intergroup differences in pain improvement at baseline, 1-year, and 2-years following rotator cuff repair $(P=$ $.110, .559, .846$, respectively). Patients in Group I improved their ASES shoulder function score by 38.5 points at 2 years after surgery and Group II improved by 41.9 points at the same time interval (Table 2 ).

\section{Postoperative Function}

All patients demonstrated improvement in shoulder function after rotator cuff repair as reported by the SANE and Simple Shoulder Test (SST) outcome measures before and after adjusting for worker's compensation status. SANE and SST outcome measures were used to assess functional disability experienced by patients because of rotator cuff tear. Both groups experienced a significant increase in SANE score: Group I improved by 44.6 points at 2 years after surgery, and Group II improved by 44.9 points at 2 years. There were no statistically significant intergroup differences in SANE score improvement over the course of the study. There were no significant intergroup differences in SST score improvement or at baseline and 1 and 2 years after operation (Table 3).

Table 4. Improvement in the VR-12M and VR-12P Ratings Across All Groups After Rotator Cuff Repair (Adjusted For Worker's Compensation Status)

\begin{tabular}{|c|c|c|c|c|}
\hline PRO & Time & No ibuprofen/NSAIDs use & Ibuprofen use & $P$ value \\
\hline VR12M & Baseline & $53.4(51.6,55.2)$ & $52.8(51.1,54.5)$ & .541 \\
\hline VR12M & Year 1 & $54.9(53.2,56.5)$ & $54.9(53.4,56.5)$ & .962 \\
\hline VR12M & Year 2 & $54.9(53.2,56.7)$ & $54.3(52.5,56.2)$ & .569 \\
\hline VR12M & Year 2 change from BL & $1.5(0.0,3.1)$ & $1.6(-0.2,3.3)$ & .987 \\
\hline VR12P & Baseline & $34.7(33.2,36.2)$ & $34.1(32.7,35.6)$ & .501 \\
\hline VR12P & Year 1 & $46.3(44.9,47.8)$ & $46.2(44.8,47.6)$ & .846 \\
\hline VR12P & Year 2 change from BL & $12.9(11.5,14.2)$ & $12.9(11.3,14.4)$ & .998 \\
\hline
\end{tabular}

BL, baseline; NSAID, nonsteroidal anti-inflammatory agent; VR-12M, Veterans Rand 12-Item Health Survey for Mental Health; VR-12P, Veterans Rand 12-Item Health Survey for Physical Health. 


\section{Overall Health Assessment}

The VR-12 assessment tool was used to assess patient's overall health. The VR-12 form consisted of a series of questions about mental, emotional, and physical health, as well as social functioning. The VR-12 is separated into a physician component score and a mental component score. The higher the score, the better the patient's perception of their overall health. Patients in both groups demonstrated improvement in the VR-12 physician component score and mental component score after rotator cuff repair. There were no statistically significant intergroup differences at baseline and 1 and 2 years after surgery. Similarly, there were no statistically significant intergroup differences in improvement at 1 and 2 years after surgery (Table 4 ).

\section{Discussion}

In this study, we found no significant difference in patient-reported outcome measures between patients who were instructed to use ibuprofen after surgery and patients who were instructed not to use NSAIDs following surgery for rotator cuff repair. This supports the hypothesis that patients would have similar reported outcomes at 1- and 2-years following arthroscopic rotator cuff repair.

Postoperative pain control is a critical component of patient care and leads to faster rehabilitation and increased function after surgical repair. There is a broad range of analgesic therapies for pain control. The traditional management of postoperative pain after rotator cuff repair is with opioid drugs, and many consider it the gold standard for analgesia after orthopedic surgery. ${ }^{17}$ Opioids also have a high potential for abuse, and thus there have been significant efforts to reduce the rate of opioids prescribed. ${ }^{35}$

Historically NSAID use has been avoided in rotator cuff repair surgery because of the potential to inhibit tendon healing. ${ }^{36}$ NSAIDs have been shown to decrease opioid requirements after surgical procedures ${ }^{24,37}$ but concern for lack of healing and inferior postoperative outcomes has prevented many surgeons from using them.

In a Level I RCT, Oh et al. ${ }^{34}$ determined there were no significant differences in pain intensity or incidence of adverse effects at 2 weeks after rotator cuff repair in individuals randomized to a selective NSAID group (celecoxib), a nonselective NSAID group (ibuprofen), or an opioid group (tramadol). However, the study determined that the selective NSAID celecoxib was correlated with a significantly higher re-tear rate compared with those taking the nonselective NSAID ibuprofen or the opioid tramadol. ${ }^{34}$

Despite the efficacy of NSAIDs in postoperative pain control, these agents are not without risk. NSAIDs reduce pain and inflammation by inhibiting cyclooxygenase activity in the arachidonic pathway, decreasing the synthesis of proinflammatory prostaglandin molecules.
The effects of NSAIDs on bone, tendon, and muscle healing have been studied in animal models and to a lesser extent in human models. Conflicting evidence exists in support of and against the use of NSAIDs in bony and tendinous healing processes. A study by Carlstedt et al. ${ }^{32}$ demonstrated the positive effect of NSAIDs on tendon healing. Carlstedt et al. ${ }^{32}$ conducted an in vitro study that examined the effects of the nonspecific NSAID indomethacin on the biomechanical properties of plantaris longus tendon healing in rabbits. The study found that indomethacin therapy significantly increased tendon tensile strength. ${ }^{32}$ Leadbetter $^{38}$ demonstrated the importance of cell proliferation, migration, and collagen synthesis for tendon repair. In animal models, Tsai et al. ${ }^{39}$ showed that NSAIDs impeded the proliferation and migration of tendon cells, delaying the healing process. Almekinders et al. ${ }^{30}$ investigated the in vitro effects of NSAIDs on human tendon fibroblasts and found that these drugs may inhibit the proliferative phase of tendon healing by impeding DNA synthesis. However, the study also found that NSAIDs stimulated protein synthesis in these same cells, demonstrating that NSAIDs may be beneficial in the remodeling phase of the healing process. ${ }^{30}$ Ferry et al. ${ }^{40}$ found that NSAIDs, with the exception of ibuprofen, had a detrimental effect on healing strength at the bone-tendon junction in animal models. A meta-analysis by Wheatley et al. $^{41}$ found that NSAID exposure delayed bone union in the adult population. These studies indicate that NSAID use plays a hindering role in tendinous and bony healing processes, but there are fewer studies that identify the direct impact of NSAID use on rotator cuff repair healing.

Despite the theoretical concerns on healing processes, there is not any strong evidence to avoid the use of NSAIDs after rotator cuff surgery. This article supports the use of ibuprofen after arthroscopic rotator cuff repair and demonstrates no difference in clinical outcomes 2 years after surgery. This should encourage surgeons to prescribe ibuprofen after surgery to potentially minimize the use of opioids.

\section{Limitations}

There are several limitations to this study. Although our patient response rate was sufficient at $80 \%$, nonresponse bias is a limitation of any retrospective review of prospectively collected data. Surgical technique was not taken into consideration, but even with variability in technique, we demonstrate that outcomes are not affected with postoperative ibuprofen use. VAS scores were collected to assess pain, but number of opioids used with or without ibuprofen was not the aim of this study, rather only patient-reported outcome measures, so we are unable to comment on whether ibuprofen use decreased opioid use after surgery. We did not assess the total consumption of ibuprofen after operation, so we cannot comment on what the patients actually consumed 
but only what was prescribed. This study looked at patient reported outcome measures only, and postoperative magnetic resonance imaging to assess healing was not performed. Also, the lack of a priori power analysis leaves the potential for a type II or beta error.

\section{Conclusion}

Patients receiving ibuprofen did as well as patients who did not receive ibuprofen after arthroscopic rotator cuff repair on patient-reported outcome measures assessing shoulder pain, function, and overall health.

\section{References}

1. Carpenter JE, Thomopoulos S, Flanagan CL, DeBano CM, Soslowsky LJ. Rotator cuff defect healing: A biomechanical and histologic analysis in an animal model. J Shoulder Elbow Surg 1998;7:599-605.

2. Castricini R, Longo UG, De Benedetto M, et al. Plateletrich plasma augmentation for arthroscopic rotator cuff repair. Am J Sports Med 201 1;39:258-265.

3. Gulotta LV. Growth factors for rotator cuff repair. Clin Sports Med 2009;28:13-23.

4. Yamaguchi K, Ditsios K, Middleton WD, Hildebolt CF, Galatz LM, Teefey SA. The demographic and morphological features of rotator cuff disease. J Bone Joint Surg 2006;88:1699-1704.

5. Chard MD, Hazleman R, Hazleman BL, King RH, Reiss BB. Shoulder disorders in the elderly: A community survey. Arthritis Rheumatism 1991;34:766-769.

6. Gumina S, Passaretti D, Candela V. Shoulder pain intensity and distribution. In: Gumina S, ed. Rotator cuff tear: Pathogenesis, evaluation and treatment. Cham, Switzerland: Springer, 2017;133-137.

7. Nakajima D, Yamamoto A, Kobayashi T, et al. The effects of rotator cuff tears, including shoulders without pain, on activities of daily living in the general population. J Orthop Sci 2012;17:136-140.

8. Vidt ME, Santago AC, Marsh AP, et al. The effects of a rotator cuff tear on activities of daily living in older adults: A kinematic analysis. J Biomech 2016;49:61 1-617.

9. Mazoue C, Andrews J. The thrower's shoulder: Management of full-thickness rotator cuff tears. Operative Tech Sports Med 2016;24:203-212.

10. Passaretti D, Candela V, Gumina S. Natural History. In: Gumina S, ed. Rotator cuff tear: Pathogenesis, evaluation and treatment. Cham, Switzerland: Springer, 2017;207-212.

11. Tashjian RZ. Epidemiology, Natural history, and indications for treatment of rotator cuff tears. Clin Sports Med 2012;31:589-604.

12. Mohana-Borges AVR, Chung CB, ResnickD. MR imaging and MR arthrography of the postoperative shoulder: Spectrum of normal and abnormal findings. RadioGraphics 2004;24:69-85.

13. Gartsman GM, Brinker MR, Khan M. Early Effectiveness of arthroscopic repair for full-thickness tears of the rotator cuff. An outcome analysis. J Bone Joint Surg 1998;80:33-40.

14. Wolf EM, Pennington WT, Agrawal V. Arthroscopic rotator cuff repair: 4- to 10-year results. Arthroscopy 2004;20: 5-12.
15. Greenspoon JA, Petri M, Warth RJ, Millet P. Massive rotator cuff tears: pathomechanics, current treatment options, and clinical outcomes. J Shoulder Elbow Surg 2015;24:1493-1505.

16. Thakkar RS, Thakkar SC, Srikumaran U, McFarland EG, Fayad LM. Complications of rotator cuff surgery - the role of post-operative imaging in patient care. Br J Radiol 2014;87:20130630.

17. Gerbershagen HJ, Aduckathil S, van Wijck AJM, Peelen LM, Kalkman CJ, Meissner W. Pain intensity on the first day after surgery. Anesthesiology 2013;118:934-944.

18. Tuominen M, Pitkänen M, Rosenberg PH. Postoperative pain relief and bupivacaine plasma levels during continuous interscalene brachial plexus block. Acta Anaesthesiol Scand 1987:31:276-278.

19. Basat HÇ, Uçar DH, Armangil M, Güçlü B, Demirtaş M. Post operative pain management in shoulder surgery: Suprascapular and axillary nerve block by arthroscope assisted catheter placement. Ind J Orthop 2016;50:584-589.

20. Boss AP, Maurer T, Seiler S, Aeschbach A, Hintermann B, Strebel S. Continuous subacromial bupivacaine infusion for postoperative analgesia after open acromioplasty and rotator cuff repair: Preliminary results. J Shoulder Elbow Surg 2014;13:630-634.

21. Ilfeld BM, Morey TE, Wright TW, Chidgey LK, Kayser Enneking F. Continuous interscalene brachial plexus block for postoperative pain control at home: A randomized, double-blinded, placebo-controlled study. Anasth Analg 2003;96:1089-1095.

22. Su B, O'Connor JP. NSAID therapy effects on healing of bone, tendon, and the enthesis. J Appl Physiol 2013;115:892-899.

23. García Rodríguez L, Jick H. Risk of upper gastrointestinal bleeding and perforation associated with individual non-steroidal anti-inflammatory drugs. Lancet 1994;343: 769-772.

24. Gupta A, Bah M. NSAIDs in the treatment of postoperative pain. Curr Pain Headache Rep 2016;20:62.

25. Allen HL, Wase A, Bear WT. Indomethacin and aspirin: Effect of nonsteroidal anti-inflammatory agents on the rate of fracture repair in the rat. Acta Orthop Scand 1980;51:595-600.

26. Elder CL, Dahners LE, Weinhold PS. A cyclooxygenase-2 inhibitor impairs ligament healing in the rat. Am J Sports Med 2001;29:801-805.

27. Simon AM, Manigrasso MB, O'Connor JP. Cyclo-oxygenase 2 function is essential for bone fracture healing. J Bone Miner Res 2002;17:963-976.

28. Zhang X, Schwarz EM, Young DA, Puzas JE, Rosier RN, O'Keefe RJ. Cyclooxygenase- 2 regulates mesenchymal cell differentiation into the osteoblast lineage and is critically involved in bone repair. J Clin Invest 2002;109:1405-1415.

29. Mallick E, Scutt N, Scutt A, Rolf C. Passage and concentration-dependent effects of Indomethacin on tendon derived cells. J Orthop Surg Res 2009;4:9.

30. Almekinders LC, Baynes AJ, Bracey LW. An in vitro investigation into the effects of repetitive motion and nonsteroidal antiinflammatory medication on human tendon fibroblasts. Am J Sports Med 1995;23:119-123.

31. Anakwenze OA, Kancherla VK, Warrender W, Abboud JA. Outcomes of modified 2-incision technique with use of indomethicin in treatment of distal biceps tendon rupture. Orthopedics $2011 ; 34: 724-729$. 
32. Carlstedt CA, Madsén K, Wredmark T. The influence of indomethacin on tendon healing. Arch Orthop Trauma Surg 1986;105:332-336.

33. DePeter KC, Blumberg SM, Dienstag Becker S, Meltzer JA. Does the use of ibuprofen in children with extremity fractures increase their risk for bone healing complications? J Emerg Med 2017;52:426-432.

34. Oh JH, Seo HJ, Lee YH, Choi HY, Joung HY, Kim SH. Do selective COX-2 inhibitors affect pain control and healing after arthroscopic rotator cuff repair? A preliminary study. Am J Sports Med 2018;46:679-686.

35. Murthy VH. Ending the opioid epidemic-A call to action. N Engl J Med 2016;375:2413-2415.

36. Tashjian RZ. Epidemiology, natural history, and indications for treatment of rotator cuff tears. Clin Sports Med 2012;31: 589-604.
37. Zhao-Fleming $\mathrm{H}$, Hand A, Zhang K, et al. Effect of nonsteroidal anti-inflammatory drugs on post-surgical complications against the backdrop of the opioid crisis. Burns Trauma 2018;6:25.

38. Leadbetter WB. Cell-matrix response in tendon injury. Clin Sports Med 1992;11:533-578.

39. Tsai WC, Hsu CC, Chou SW, Chung CY, Chen J, Pang JHS. Effects of celecoxib on migration, proliferation and collagen expression of tendon cells. Connect Tissue Res 2007;48:46-51.

40. Ferry ST, Dahners LE, Afshari HM, Weinhold PS. The effects of common anti-inflammatory drugs on the healing rat patellar tendon. Am J Sports Med 2007;35:1326-1333.

41. Wheatley BM, Nappo KE, Christensen DL, Holman AM, Brooks DI, Potter BK. Effect of NSAIDs on bone healing rates. J Am Acad Orthop Surg 2018;27:1. 